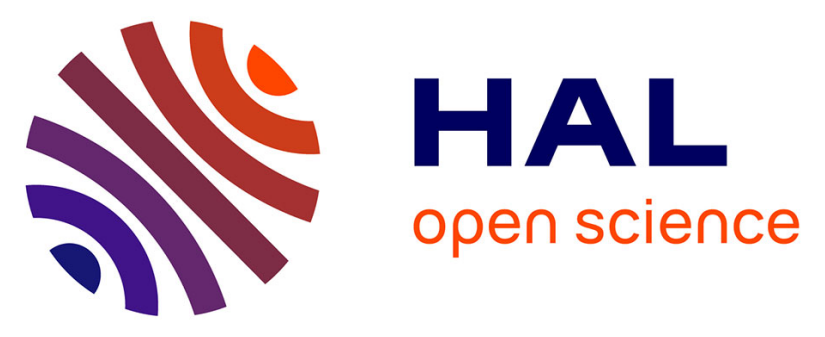

\title{
Titanium dioxide waveguides for supercontinuum generation and optical transmissions in the near-and mid-infrared
}

Manon Lamy, Christophe Finot, Laurent Markey, Juan Arocas, Julien Fatome, Bertrand Kibler, Alain Dereux, Jean-Claude Weeber, Kamal Hammani

\section{To cite this version:}

Manon Lamy, Christophe Finot, Laurent Markey, Juan Arocas, Julien Fatome, et al.. Titanium dioxide waveguides for supercontinuum generation and optical transmissions in the near-and midinfrared. 21th International Conference on Transparent Optical Network (ICTON), Jul 2019, Angers, France. pp.Th.A1.4. hal-02190034

\section{HAL Id: hal-02190034 \\ https://hal.science/hal-02190034}

Submitted on 21 Jul 2019

HAL is a multi-disciplinary open access archive for the deposit and dissemination of scientific research documents, whether they are published or not. The documents may come from teaching and research institutions in France or abroad, or from public or private research centers.
L'archive ouverte pluridisciplinaire HAL, est destinée au dépôt et à la diffusion de documents scientifiques de niveau recherche, publiés ou non, émanant des établissements d'enseignement et de recherche français ou étrangers, des laboratoires publics ou privés. 


\title{
Titanium dioxide waveguides for supercontinuum generation and optical transmissions in the near- and mid-infrared
}

\author{
Manon Lamy, Christophe Finot, Laurent Markey, Juan Arocas, \\ Julien Fatome, Bertrand Kibler, Alain Dereux, Jean-Claude Weeber, and Kamal Hammani \\ Laboratoire Interdisciplinaire Carnot de Bourgogne (ICB), UMR 6303 CNRS - Université Bourgogne Franche- \\ Comté, 9 avenue Alain Savary, BP 47870, 21078 Dijon Cedex, France \\ e-mail: Kamal.Hammani@u-bourgogne.fr
}

\begin{abstract}
We report the development of titanium dioxide-based waveguides for applications in the near- and midinfrared. Thanks to embedded metal grating couplers, we demonstrate error free $10 \mathrm{Gbit} / \mathrm{s}$ optical transmissions at 1.55 and $2 \mu \mathrm{m}$. With additional management of the dispersion profile, we also demonstrate octave spanning supercontinuum in cm-long $\mathrm{TiO}_{2}$ waveguides.
\end{abstract}

Keywords: Titanium Dioxide waveguides, Integrated optical materials, Optical Communications, Supercontinuum generation, Nonlinear integrated optics

\section{INTRODUCTION}

Since the 1980s, it becomes clear that the speed of electronics may ultimately limit the information capacity. In the context of the quest for all-optical transparent transmissions, this has stimulated many researches in the field of integrated photonic as an alternative to electronic circuits, enabling low footprint and reduced power consumption. The Kerr nonlinearity of optical material may also be a key advantage to optically process data streams and to achieve, for example, on the chip wavelength conversion.

Most of the efforts have focused on the silicon-on-insulator technology (SOI). The choice of SOI has been driven by the high nonlinearity of silicon [1] and its compatibility with low-cost large-scale production using CMOS infrastructure. However, its strong two photon absorption (TPA) in the conventional band of optical transmissions may remain an issue. Therefore, to overcome such a stringent limitation, other nonlinear platform deserve attention and various materials have been investigated such as silicon germanium [2] or silicon nitride [3], III-V material or chalcogenides [4]. Each material provides specific advantages but may also suffer from strong potential drawbacks so that the choice of a material often results from a trade-off between several criteria.

Here, we plan to explore another platform: the titanium dioxide $\left(\mathrm{TiO}_{2}\right)$. This material, well known for its photocatalytic properties or its performance for solar cells, remains largely unexplored in the field of nonlinear photonics and optical transmissions. Compared to silicon nitride, $\mathrm{TiO}_{2}$ exhibits similar nonlinear index $(\sim 0.2 \mathrm{x}$ $10^{-18} \mathrm{~m}^{2} / \mathrm{W}$ ) but a higher linear refractive index (typically 2.4 versus 2.0 for $\mathrm{Si}_{3} \mathrm{~N}_{4}$ ). This enables stronger confinement and therefore leads to higher effective nonlinearities. $\mathrm{TiO}_{2}$ also benefits from an easier deposition process and is TPA-free. We demonstrate here that this platform is fully suitable for optical telecommunication, both in the C-band of optical telecommunications and around $2 \mu \mathrm{m}$. We also definitively confirm the strong nonlinear potential by achieving an octave spanning supercontinuum.

\section{TITANIUM DIOXIDE STRUCTURES FOR OPTICAL COMMUNICATIONS}

In this first part, we present titanium dioxide waveguides operating at the conventional wavelength of $1.55 \mu \mathrm{m}$. We also explore the performance of these passive components around $2 \mu \mathrm{m}$. Indeed, this spectral window currently stimulates large experimental interest due to its potential use as a solution to the capacity crunch [5] : platforms such as $\mathrm{Si}$, SRN, SiGe have been recently successfully tested in the context of high speed optical telecommunications [6,7]. After having described the design of the $\mathrm{TiO}_{2}$ devices we detail experimental set-ups and validation of the components for error-free transmission of $10 \mathrm{~Gb} / \mathrm{s}$ on-off keying signals.

\subsection{Design and fabrication of the couplers and the waveguides}

Whatever the platform used, a crucial step is always to efficiently inject the light into the device. Basically, there are two main ways to couple light from an optical fiber to a photonic waveguide: the end-fire coupling (also known as butt-coupling) and grating coupling. Thanks to the ease of $\mathrm{TiO}_{2}$ deposition, we have been able to consider embedded metal grating couplers (Fig. 1(a)) allowing etching free process. Such metal grating couplers have been recently proposed to demonstrate high coupling efficiencies [8]. Unlike previous studies, we considered a metal 
(Au) grating buried between two $\mathrm{TiO}_{2}$ layers on a glass substrate. This provides an additional degree of freedom and lead us to a better coupling efficiency [9].

We optimized the geometric parameters with 2D numerical simulations using a commercial finite elementbased software, namely COMSOL Multiphysics, to target the best performance, around $1.55 \mu \mathrm{m}$ wavelength on one side and around $2.0 \mu \mathrm{m}$ on the other side, with an incident angle of $30^{\circ}$ fixed by the experimental set-up constraints. The simulated source has a TM polarization for the considered embedded gratings. For the two considered spectral bands, the best set of parameters is obviously different.

Then the devices have been fabricated combining widely-spread techniques such as reactive DC magnetron sputtering to deposit titanium dioxide layers, electron beam lithography, thermal evaporation and reactive-ion etching. The resulting devices have the following measured parameters, respectively at $1.55 \mu \mathrm{m}$ and at $2 \mu \mathrm{m}$ : a top layer thickness $h_{\text {top }}$ of $189 \mathrm{~nm}$ and $235 \mathrm{~nm}$, a grating period $\Lambda$ of $1375 \mathrm{~nm}$ and $1900 \mathrm{~nm}$ and a grating line width $\mathrm{w}_{1}$ of about $610 \mathrm{~nm}$ and $630 \mathrm{~nm}$. For the whole devices, the bottom layer thickness $\mathrm{h}_{\text {bottom }}$ is $69 \mathrm{~nm}$ and the gold thickness $\mathrm{h}_{\mathrm{Au}}$ is $56 \mathrm{~nm}$.

\subsection{Test of the devices coupling efficiency}

Before transmitting an optical signal in the titanium dioxide waveguides, we have measured their total losses. The structures under test should ideally present a good total coupling efficiency to enable high speed optical transmissions at $1.55 \mu \mathrm{m}$ and $1.98 \mu \mathrm{m}$. Their losses are measured with a set-up composed of two optical focusers based on lensed fibers. IR and visible cameras enable us to adjust the focusers onto the grating couplers to optimize the injection and collection of the light. A powermeter and optical spectrum analyser are also used us to optimize the focusers adjustment and record the output light.

At $1550 \mathrm{~nm}$, we have measured losses as low as - 8dB per facet with a $-3 \mathrm{~dB}$ transmission bandwidth covering the whole C-band with a fair agreement with numerical simulations as shown in Fig. 1(b). Around $2 \mu \mathrm{m}$, the coupling losses per facet reach $-10 \mathrm{~dB}$. Note that for this wavelength, the component parameters have been chosen so that TE and TM polarisation exhibit similar performance. The difference between the numerical simulations and the efficiency measured experimentally can be in a large extend assigned to discrepancies between the targeted values and the values reached at the end of the various fabrication processes. Future improvements may involve metal mirror under the gratings or parabolic gratings. However, as we will see, the coupling efficiencies are already sufficient for the transmission of a coded signal.

\subsection{Transmission at $1.55 \mu \mathrm{m}$ and at $1.98 \mu \mathrm{m}$}

For both wavelengths, a $10 \mathrm{~Gb} / \mathrm{s}$ signal coded through a $2^{31}$ pseudo random bit sequence (PRBS) generator is transmitted through the waveguides. A bit error tester or a high speed sampling oscilloscope to image eye diagrams are used as diagnostic tools of the transmission quality (show in insets of Fig. 1(b) and (c)).

At $1.55 \mu \mathrm{m}$, we used standard Small Form Factors pluggable emitters and transmitters and no optical amplifier was involved in the setup. Figure 1(b) shows wide-opened eye diagrams meaning a transmission without any degradation confirmed by an error free BER measurement (not shown here). We have also tested that our component could sustain the simultaneous transmission of several coarse wavelength-division multiplexed channels without major impairments in the C-band that corresponds to the design of our grating. Co- and counterpropagating schemes were investigated.
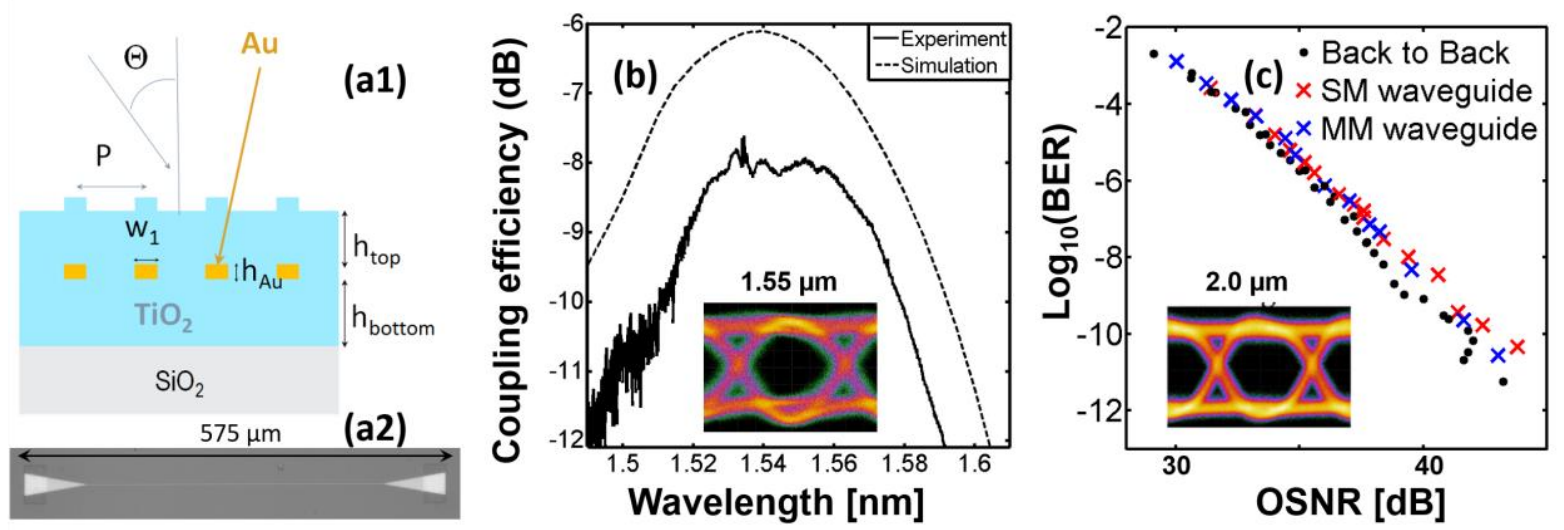

Figure 1. (a1) sketch of the embedded metal grating in titanium dioxide layer (side view). (a2) Top view of $1.5 \mu$ m-wide waveguide with visible camera. (b) Coupling efficiency per facet of embedded grating coupler as a function of the injected wavelength. The experimental results are compared with numerical simulations. The inset shows eye diagram of $10 \mathrm{~Gb} / \mathrm{s}$ signal transmitted into the waveguide. (c) BER measurement as a function of the OSNR. The inset shows the typical eye diagram after the waveguide at $2 \mu m$. 
At $2 \mu \mathrm{m}$, the initial signal is obtained using a Lithium-Niobate modulator that is further amplified using a Thulium-doped fiber amplifier. We have tested multimode (MM) and singlemode (SM) waveguides with a length of half a millimetre. The widths of the waveguides are 8.0 and $1.6 \mu \mathrm{m}$ respectively. In both cases, no degradation are visible on the eye diagram and BER measurements with the presence of the waveguide are very similar to the one obtained in the back-to-back configuration [10].

\section{TITANIUM DIOXIDE WAVEGUIDES FOR SUPERCONTINUUM GENERATION}

To become an interesting photonic platform for nonlinear functionalities, a material has to exhibit a strong Kerr coefficient. A way to stress the nonlinear potential of $\mathrm{TiO}_{2}$ waveguides is to evaluate its potential in the context of supercontinuum generation [11].

Contrary to previous waveguides, here we consider thicker $\mathrm{TiO}_{2}$ layers of $450 \mathrm{~nm}$ that are deposited on a 2" silicon wafer, covered with $2 \mu \mathrm{m}$ of silica, still by reactive direct current magnetron sputtering of a $99.9 \%$ pure titanium target under argon and oxygen-controlled atmosphere. Then, $2.2 \mathrm{~cm}$-long ridge waveguide having about $1.5 \mu \mathrm{m}$ is patterned thanks to an UV lithography. After the reactive ion etching, the residual mask is stripped from the waveguides. Details of the structure used in the following experiments are presented in Fig. 2(a) as well as the dispersion profile of the fundamental TE mode (Fig. 2(b)). Indeed, the transverse dimensions of the waveguide have to be carefully optimized to provide the anomalous dispersion required to achieve the largest spectral extend.

The input pulses are provided by a femtosecond laser source centered at $1560 \mathrm{~nm}$ with a peak power up to a few tens of kW. Due to the length of standard anomalous fiber between the fs-laser output and the $\mathrm{TiO}_{2}$ waveguide, the central wavelength of the pulse is shifted toward $1640 \mathrm{~nm}$. In order to optimize the light injection through buttcoupling, two 1-mm long tapers have been included in the $\mathrm{TiO}_{2}$ device. The broadest spectrum we obtained is plotted in Fig. 2(c1) along with the input pump spectrum. An octave-spanning supercontinuum is generated from $1050 \mathrm{~nm}$ up to $1910 \mathrm{~nm}$, which represents an improvement by much more than one order of magnitude compared to [12]. The nonlinear dynamics governing the spectral broadening of the pulse is mainly governed by soliton selffrequency shift and the emergence of dispersive waves in the vicinity of the zero-dispersion wavelengths. The third harmonic is also generated into the waveguide so that visible light is also clearly observed by the naked eye as can be seen from the picture taken from the top of the waveguide (Fig. 2(c2)).
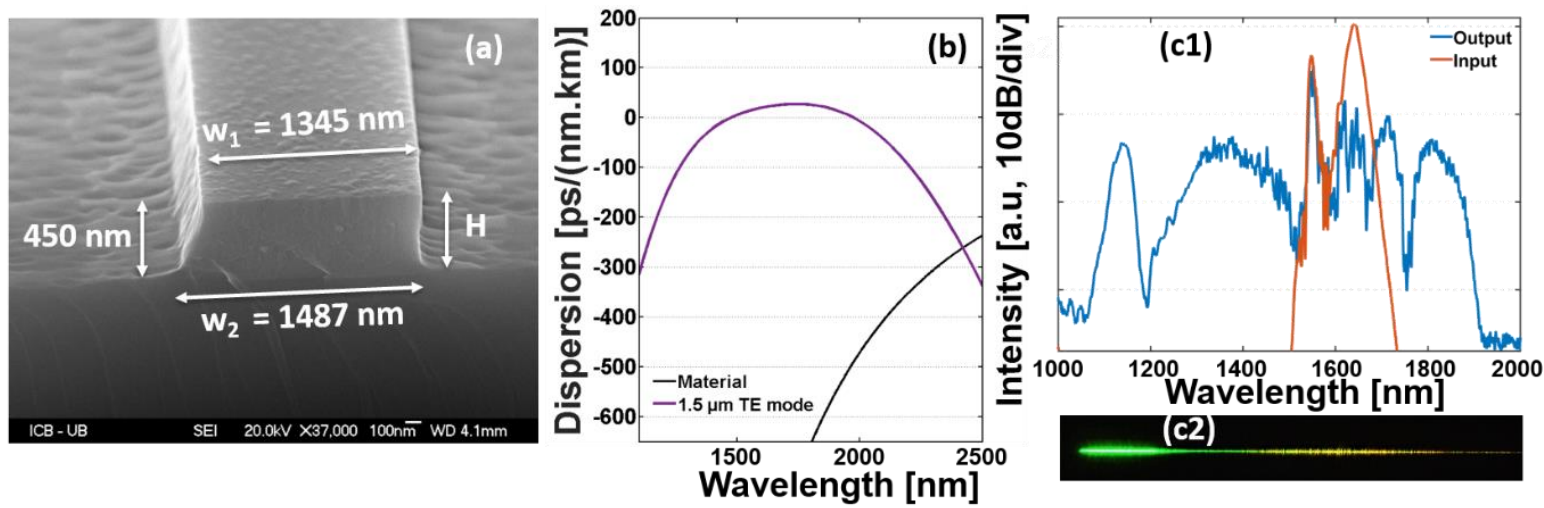

Figure 2. (a) Scanning Electron Microscope image of the cleaved strip waveguide (b) Dispersion properties for the fundamental $\mathrm{TE}_{0,0}$ mode. (c1) Optical supercontinuum obtained at the output of the waveguide (solid blue line) compared to the input pulse spectrum (red solid line). (c2) Image of the emitted visible light from the waveguide (total length $~ 6.5 \mathrm{~mm}$ ).

\section{CONCLUSIONS}

We have demonstrated error-free transmission of high bit rate signals through titanium dioxide waveguides. A new design of embedded metal grating has been tested for coupling / decoupling the signal in the C-band as well as around $2 \mu \mathrm{m}$. Such embedded configuration was made possible thanks to the ease of fabrication of this material. BER measurements of $10 \mathrm{Gbps}$ signals are confirmed the suitability of the material for optical communication and the possibility to achieve components with subwavelength transverse dimensions. In order to confirm the nonlinear potential of the $\mathrm{TiO}_{2}$ and the tailoring of its dispersive properties, we have also reported the first experimental demonstration of an octave spanning supercontinuum. 
These studies clearly demonstrate that titanium dioxide can be an alternative platform for integrated photonic. However, to date, the main drawback remains the propagation losses. We are currently working on different ways to improve the fabrication mainly regarding the etching that has been identify as the main origin of losses since it causes strong roughness of sidewall [13].

\section{ACKNOWLEDGEMENTS}

This work was supported by the European Union within the framework of the operational Program FEDERFSE Bourgogne 2014/2020, by the Région Bourgogne (Pari Photcom), by the Institut Universitaire de France and by the French "Investissements d'Avenir" program, project ISITE-BFC (contract ANR-15-IDEX-0003).

\section{REFERENCES}

[1] J. Leuthold, C. Koos, and W. Freude, "Nonlinear silicon photonics," Nature Photonics, vol. 4, no. 8, pp. 535$544,2010$.

[2] K. Hammani et al., "Optical properties of silicon germanium waveguides at telecommunication wavelengths,", Opt. Express, vol. 21, no. 14, pp. 16690-16701, 2013.

[3] M. A. G. Porcel et al., "Two-octave spanning supercontinuum generation in stoichiometric silicon nitride waveguides pumped at telecom wavelengths," Optics Express, vol. 25, no. 2, pp. 1542-1554, 2017.

[4] M. R. E. Lamont, B. Luther-Davies, D.-Y. Choi, S. Madden, and B. J. Eggleton, "Supercontinuum generation in dispersion engineered highly nonlinear $(\gamma=10 / \mathrm{W} / \mathrm{m})$ As2S3 chalcogenide planar waveguide," Optics Express, vol. 16, no. 19, pp. 14938-14944, 2008.

[5] F. Garcia Gunning, N. Kavanagh, E. Russell, R. Sheehan, J. O'Callaghan, and B. Corbett, "Key Enabling Technologies for Optical Communications at 2000 nm," Appl. Opt., vol. 57, no. 22, pp. E64-E70, 2018.

[6] M. Lamy et al., "Silicon Waveguides for High-Speed Optical Transmissions and Parametric Conversion around $2 \mu \mathrm{m}$," IEEE Photon. Technol. Lett., vol. 31, no. 2, pp. 165-168, 2019.

[7] M. Lamy et al., "Ten gigabit per second optical transmissions at $1.98 \mu \mathrm{m}$ in centimetre-long SiGe waveguides," , Electron. Lett., vol. 53, no. 17, pp. 1213-1214, 2017.

[8] S. Scheerlinck, J. Schrauwen, F. Van Laere, D. Taillaert, D. Van Thourhout, and R. Baets, "Efficient, broadband and compact metal grating couplers for silicon-on-insulator waveguides,", Opt. Express, vol. 15, no. 15, pp. 9625-9630, 2007.

[9] M. Lamy, K. Hammani, J. Arocas, C. Finot, and J. Weeber, "Broadband metal grating couplers embedded in Titanium dioxide waveguides " Opt. Lett., vol. 42, no. 14, pp. 2778-2781, 2017.

[10] M. Lamy, C. Finot, J. Fatome, J. Arocas, J.-C. Weeber, and K. Hammani, "Demonstration of High-Speed Optical Transmission at $2 \mu \mathrm{m}$ in Titanium Dioxide Waveguides," Applied Sciences, vol. 7, no. 6, p. 631, 2017.

[11] K. Hammani et al., "Octave Spanning Supercontinuum in Titanium Dioxide Waveguides," Applied Sciences, vol. 8, no. 4, p. 543, 2018.

[12] C. C. Evans, K. Shtyrkova, J. D. B. Bradley, O. Reshef, E. Ippen, and E. Mazur, "Spectral broadening in anatase titanium dioxide waveguides at telecommunication and near-visible wavelengths," Opt. Express, vol. 21 , no. 15 , pp. 18582-18591, 2013.

[13] C. C. Evans, C. Liu, and J. Suntivich, "Low-loss titanium dioxide waveguides and resonators using a dielectric lift-off fabrication process," Opt. Express, vol. 23, no. 9, pp. 11160-11169, 2015. 